\title{
Geometria e funções: uma abordagem computacional a partir de problemas da OBMEP
}

\section{Geometry and functions: a computational approach based on OBMEP problems}

\author{
Aline de Lima Guedes Machado \\ Universidade do Estado do Rio de Janeiro (UERJ), Departamento de Matemática Aplicada \\ Rio de Janeiro, RJ, Brasil \\ aline.guedes@ime.uerj.br \\ Leandro da Silva Machado \\ Universidade do Estado do Rio de Janeiro (UERJ) \\ Instituto de Aplicação Fernando Rodrigues da Silveira/CAp-UERJ, Rio de Janeiro, RJ, Brasil \\ leandromachado@ime.ueri.br
}

\begin{tabular}{|l} 
Informações do Artigo \\
\hline cc) () \& \\
\hline $\mathrm{BY}$
\end{tabular}

Histórico do Artigo

Submissão: 30 de agosto de 2018 .

Aceite: 22 de novembro de 2018.

Palavras-chave

Funções Reais

Geométrico-funcional

Geometria Dinâmica

OBMEP

Educação Básica

\section{Resumo}

Neste trabalho, propomos uma reflexão sobre a integração dos campos algébrico e geométrico no ensino de funções reais nas aulas de Matemática da Educação Básica, através de atividades desenvolvidas num ambiente de geometria dinâmica. Foi feito um recorte com problemas recorrentes da OBMEP, entre 2005 e 2017, que apresentam esse cunho geométrico-funcional. A metodologia está focada na elaboração de roteiros de atividades no Geogebra, a fim de garantir que o conceito de função também seja compreendido como uma relação de dependência entre objetos geométricos, contribuindo assim para expandir as imagens de conceito e as relações existentes entre funções reais e geometria.

\section{Keywords}

Real Functions

Geometric-functional

Dynamic Geometry

OBMEP

Basic Education

\begin{abstract}
This paper presents a proposal for reflection on the integration of algebraic and geometric fields in real functions teaching in Mathematics classes, through activities developed in a dynamic geometry environment. A cut-out was made with recurrent problems in the OBMEP, from 2005 to 2017, which present this geometric-functional character. The methodology is focused on the elaboration of activities guides in Geogebra, in order to ensure that the concept of function is also understood as a relation of dependence between geometric objects, thus contributing to expand the concept images and the existing relations between real functions and geometry.
\end{abstract}

\section{Introdução}

Fazer Matemática nos dias de hoje vai muito além de simplesmente dominar fórmulas e procedimentos para a resolução de problemas, como destacam Gravina e Santarosa (1998), Pavanello e Nogueira (2006) e Meneghetti e Redling (2012). É importante e necessário que a Matemática também contribua para o desenvolvimento do raciocínio lógico, permitindo não só a resolução correta de um problema, mas também a construção de uma argumentação sobre diferentes soluções, além da capacidade de analisar estratégias, formar conjecturas e estabelecer 
conexões do que está sendo estudado com outros saberes, tanto dentro como fora da Matemática (BRASIL, 2002; 2017).

Desta forma, as aulas de Matemática devem proporcionar um ambiente que estimule a reflexão, com atividades que conduzam a diferentes caminhos para a solução de um mesmo problema. Além disso, é importante que sejam utilizadas estratégias pedagógicas diferenciadas para apresentar e desenvolver um determinado conteúdo. O desenvolvimento do raciocínio lógico, através da resolução de problemas e investigação de resultados, é destacado nos PCNs (2002):

Por exemplo, é bem diferente a natureza das competências envolvidas na solução de um dado problema em que é apenas solicitado o cálculo da distância percorrida por um corpo com desaceleração constante, e de um outro em que se solicita a análise das consequências de altas velocidades de veículos. Embora nessas duas situações a solução do problema exija o mesmo instrumental matemático, a própria estratégia para a resolução de problemas é também diferente. Enquanto na primeira trata-se de associar os elementos do enunciado a uma equação matemática, já na segunda são necessários a identificação da situação-problema, o levantamento de hipóteses, a escolha de caminhos para a solução, além da análise dos resultados, principalmente no que diz respeito à sua coerência com o que o aluno conhece da realidade. (BRASIL, 2002, p. 85).

Atrelada a essa necessidade do estímulo de atividades que contribuam para o desenvolvimento de argumentações e levantamento de hipóteses, é grande a relevância da interrelação entre conteúdos dentro da própria Matemática que, por diversos motivos, acabam sendo trabalhados de formas isoladas em diferentes setores, sem articulação entre eles. Cabe ressaltar que os documentos oficiais (BRASIL, 2017) estimulam essa inter-relação entre conteúdos:

Inter-relações devem ser destacadas, por exemplo, entre a Estatística e a Probabilidade, a Álgebra e a Geometria. A própria ideia de medida pode ser definida como uma função que associa um número real positivo [...] a um comprimento, área ou volume. (BRASIL, 2017, p. 521).

Desta forma, é possível afirmar que a integração dos diferentes campos da Matemática algébrico, aritmético e geométrico - se faz necessário nas salas de aula. Selecionamos o conceito de função real para este trabalho, uma vez que ele se faz presente em diversas áreas do conhecimento, com conexões dentro e fora da própria Matemática. Além disso, como ressaltam Lima et al. (1996), o conceito de função pode ser considerado um dos pilares da Matemática:

[...] os conjuntos são o modelo matemático para a organização do pensamento lógico; os números são o modelo para as operações de contagem e medida; as funções [...] cada uma delas é estudada como modelo adequado para representar uma situação específica. (LIMA et al., 1996).

A principal essência da definição do conceito de função é a relação de dependência entre duas grandezas (HAZZAN; GOLDENBERG, 1997, REZENDE et al., 2011, NEVES; RESENDE, 2016, SANTOS; BARBOSA, 2017). Entretanto, muito destaque é dado para se encontrar a lei da função que modela um problema proposto (MENEGHETTI; REDLING, 2012, SANTOS; BARBOSA, 2017), em que realmente uma grandeza depende da outra (já que caso contrário não teria sentido o uso de funções), ficando em menor destaque esta relação de dependência em si, no sentido de avaliar como a variação de uma dessas grandezas depende da variação da outra grandeza. Além disso, é possível afirmar, com uma rápida pesquisa em livros didáticos e em questões de concursos 
anteriores, como vestibulares, ENEM e mesmo em concursos para professores de Matemática, que é dada uma ênfase maior em problemas algébricos ou aritméticos, em detrimento de problemas geométricos com apelo funcional. Ou ainda, quando problemas geométricos são apresentados, o foco é o cálculo de valores máximos ou mínimos da função que modela o problema em questão e não a análise das variações das grandezas envolvidas.

A importância e a relevância da relação geométrica-funcional são apontadas em diversos estudos como, por exemplo, em Hazzan e Goldenberg (1997), Stormowski et al. (2015), Bifano e Lupinacci (2012), Giraldo (2012) e Machado (2015). Entretanto, apesar dos estímulos apresentados nos documentos oficiais (BRASIL, 2002;2017) e nas pesquisas científicas citadas, essa relação ainda é pouco explorada. Na maioria das vezes, encontramos a separação dos conteúdos com questões exclusivamente funcionais e/ou exclusivamente geométricas.

No presente trabalho, apresentamos uma proposta diferenciada dos livros didáticos usuais, baseando-nos em questões da Olimpíada Brasileira de Matemática das Escolas Públicas (OBMEP) que apresentam esse cunho geométrico-funcional. A relevância da OBMEP tem sido comprovada pela crescente adesão de escolas públicas e privadas dos Ensinos Fundamental e Médio, em todo Brasil, indicando que suas questões podem e devem ser trabalhadas em salas de aulas regulares.

Portanto, nosso objetivo é propor uma reflexão sobre a integração dos campos algébrico e geométrico no ensino de funções reais nas aulas de Matemática da Educação Básica, através de atividades desenvolvidas num ambiente de geometria dinâmica e, para isto, faremos um recorte com problemas recorrentes na OBMEP, que apresentam esse cunho geométrico-funcional. Além disso, discutimos acerca da relevância de se trabalhar diferentes contextos para tratar um determinado assunto, com o objetivo de ampliar, de maneira significativa, as imagens de conceito (TALL e VINNER, 1981), em particular o conceito de funções reais.

\section{OBMEP}

A Olimpíada Brasileira de Matemática das Escolas Públicas (OBMEP) é uma competição científica, considerada como um projeto nacional de política pública de educação, realizada desde 2005 pelo Instituto Nacional de Matemática Pura e Aplicada (IMPA) em parceria com a Sociedade Brasileira de Matemática (SBM) e com apoio financeiro do Ministério da Educação (MEC) e do Ministério da Ciência, Tecnologia, Inovações e Comunicações (MCTIC).

Ao longo destes mais de 10 anos de existência, é possível perceber alguns dos efeitos positivos gerados por esta política pública (MEZA; SCHIRLO, 2013), que visa estimular e promover o estudo da Matemática, como os impactos nas escolas públicas e um melhor desempenho em avaliações brasileiras (CGEE, 2011, SOARES et al., 2014). Além disso, números extremamente relevantes, como a presença em $99,44 \%$ dos municípios brasileiros e os mais de 18 milhões de 
alunos inscritos (dos Ensinos Fundamental e Médio) para a edição de $2018^{1}$ mostram sua dimensão. Contando também com alunos da rede privada de ensino, admitidos desde 2017, a competição foi estendida para alunos dos $4^{\circ}$ e $5^{\circ}$ anos do Ensino Fundamental em 2018.

Diante do exposto, é natural que a OBMEP venha se tornar objeto de estudo. Desta forma, propusemos um estudo acerca de um tipo de problema recorrente nas edições da OBMEP, entre 2005 e 2017, tanto em suas primeiras fases (questões objetivas) quanto nas segundas (questões dissertativas): problemas de natureza geométrica nos quais existe um comportamento funcional entre dois determinados parâmetros. Um exemplo é dado pela Figura 1.

Figura 1 - Problema Selecionado 1: OBMEP 2007, $1^{\text {a }}$ Fase, Nível 3, Questão 18.

Qual dos gráficos abaixo descreve a variação da área do poligono $\mathrm{BCDP}$ em função da distância $\mathrm{x}=\mathrm{AP}$ ?
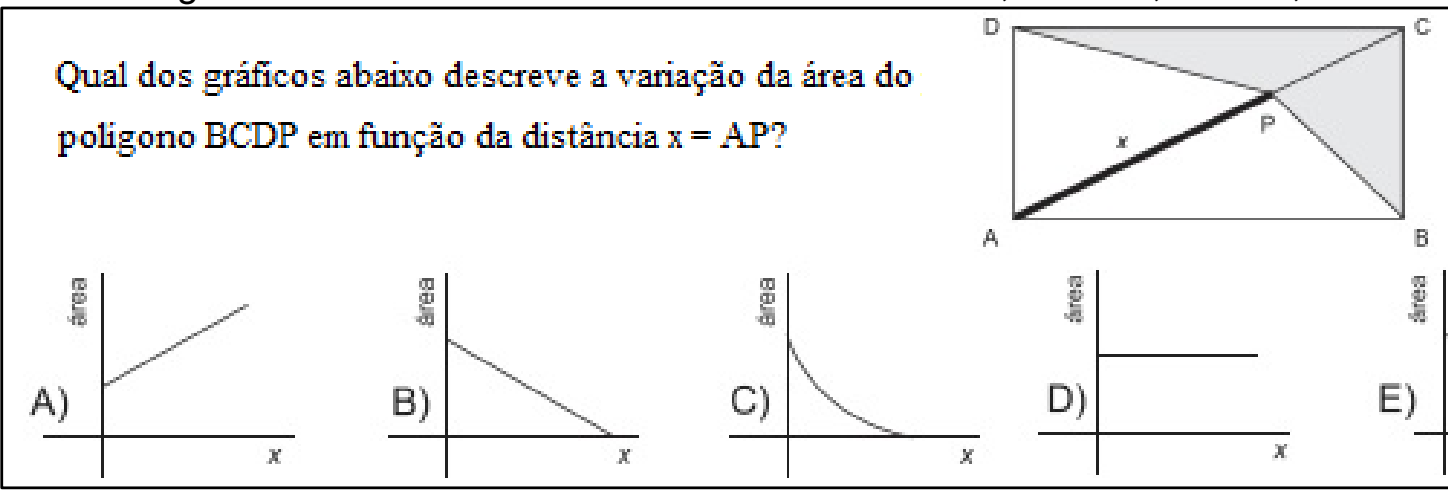

E)

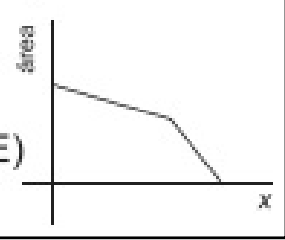

Fonte: OBMEP (2007, p. 4).

A escolha deste tipo de problema pode ser considerada relevante devido a alguns fatoreschave: a área "Geometria", historicamente, é a que traz maior dificuldade aos alunos do Ensino Básico (HARTWING et al., 2016); o tema "Funções" é o objeto de estudo no final do Ensino Fundamental e está em foco durante todo o Ensino Médio (BRASIL, 2002; 2017) e a própria especificidade deste tipo de problema nos permite fazer uma abordagem computacional, através de um software de geometria dinâmica como o "Geogebra", integrando o aproveitamento de tecnologias digitais ao ensino da Matemática, que é tão estimulado (BRASIL, 2002; 2017).

\section{Funções Reais e Imagem de Conceito}

Apesar das definições desempenharem um papel fundamental na Matemática, a definição de um objeto matemático pode não concentrar, por si só, todas as características que esse objeto possa refletir. Por exemplo, uma definição usual de função real é apresentada no Quadro 1.

Entretanto, só esta definição de função real não permite avaliar todo o potencial inerente a este conceito. Outras características podem ser consideradas para dar uma ideia mais abrangente ou mais objetiva, de acordo com a necessidade em que o conceito funcional será aplicado, como, por exemplo, dependência, relação, transformação, variação ou generalização. Além disso, a

\footnotetext{
${ }^{1}$ Números obtidos na página oficial da OBMEP. Disponível em: http://www.obmep.org.br. Acesso em: 30 ago. 2018.
} 
compreensão dessas características pode variar de acordo com cada percepção individual, podendo ainda ser mutável ao longo do tempo ou após novas experiências com a Matemática.

Quadro 1 - Definição formal de função real.

Sejam $X$ e $Y$ dois conjuntos quaisquer. Uma função é uma relação $f: X \rightarrow Y$ que, a cada elemento $x \in X$, associa um e somente um elemento $y \in Y$. Além disso,

(i) Os conjuntos $X$ e $Y$ são chamados de domínio e contradomínio de f, respectivamente;

(ii) O conjunto $f(X)=\{y \in Y ; \exists x \in X, f(x)=y\} \subset Y$ é chamado de imagem de $\mathrm{f}$;

(iii) Dados $x \in X$, o (único) elemento $y=f(x) \in Y$ correspondente é chamado de imagem de $x$.

Fonte: Lima (2013, p. 3).

Tall e Vinner (1981) desenvolveram uma teoria sobre "Imagem de Conceito", que trata exatamente sobre essa discussão acerca da apresentação até a verdadeira internalização de um conceito matemático em si. De acordo com os autores, quando alguém é levado a refletir sobre um assunto, em particular sobre um objeto matemático, a mente é estimulada a trabalhar e com isso várias representações mentais podem surgir, como: propriedades, formalizações, imagens representativas, percepções individuais e vivências. A esse conjunto de representações mentais, que ressaltamos ser um conjunto individual e que pode variar de indivíduo para indivíduo, de realidade para realidade, os autores definem como "Imagem de Conceito":

[...] estrutura cognitiva total que está associada ao conceito, que inclui todas as imagens mentais, propriedades e processos associados. Ela é construída ao longo dos anos, através de experiências de todos os tipos, mudando à medida que o indivíduo encontra novos estímulos e amadurece. (TALL e VINNER, 1981, p. 152, tradução nossa).

Aplicando esta teoria ao falar sobre a "Imagem de Conceito" do objeto matemático "Função Real" para um determinado indivíduo, todos os atributos que ele possui em sua mente sobre esse conceito vão surgindo à medida em que é estimulado a refletir. Pode-se destacar como representações mentais: gráficos, tabelas, dependência entre grandezas, relações entre conjuntos, tipos particulares de funções ou fórmulas. Neste contexto, "[...] a imagem de conceito de um indivíduo não é uma estrutura estática, ela sofre transformações de acordo com o desenvolvimento cognitivo do sujeito, podendo ter atributos incluídos, excluídos ou modificados" (GIRALDO, 2004).

Desta forma, o enfoque pedagógico sugerido para tratar um conceito matemático a partir da teoria aqui discutida deve, primordialmente, estimular a construção de conexões múltiplas dentro (e fora) da própria Matemática, contemplando a maior quantidade possível de imagens de conceito.

Assim, com relação ao ensino de funções reais, sugere-se propor uma ampla quantidade de abordagens e representações, com discussões que perpassam por diferentes direções como sugerem, por exemplo, Hazzan e Goldenberg (1997), Gravina et al. (2015), Neves e Resende (2016) e Santos e Barbosa (2017): função como relação de dependência; tabela de dados; máquina de transformação; expressão algébrica; gráficos; relação entre conjuntos; definição e função em 
ambientes computacionais. Neste trabalho, destaca-se uma outra abordagem: "função como relação de dependência entre objetos geométricos".

Cabe destacar que este tipo de abordagem, com cunho geométrico-funcional, já se faz presente na pesquisa científica há algum tempo (HAZZAN; GOLDENBERG, 1997, BIFANO; LUPINACCI, 2012; GIRALDO, 2012, GRAVINA et al., 2015 e MACHADO, 2015). Hazzan e Goldenberg (1997) estudaram como o conceito de função pode ser ampliado sob a perspectiva de um ambiente de geometria dinâmica e propuseram atividades cujo objetivo era uma exploração qualitativa do conceito, sem referências formais e baseadas em investigações sobre onde se encontram as relações funcionais de dependência, quais grandezas são tratadas como variáveis dessas funções e quais propriedades dessas funções podiam ser reconhecidas, entre outros:

[...] o que se entende pelo "comportamento" de uma função não é mais um rótulo verbal que se abstrai analiticamente de várias formas estáticas (símbolos, tabelas ou gráficos): tem a natureza ativa à qual nós associamos com a palavra "comportamento" em outros campos, e é diretamente observável e aberta para experimentação (HAZZAN; GOLDENBERG, 1997, p. 269, tradução nossa).

Eles destacam que é comum os alunos terem maior facilidade na realização de atividades que envolvam uma avaliação numérica ou alguma manipulação algébrica das funções, mas tendem a encontrar dificuldades em analisar ou até mesmo descrever o comportamento funcional. Desta forma, ao dar a oportunidade para que os alunos trabalhem em diferentes contextos nos quais exista o aspecto funcional, em particular nos ambientes de geometria dinâmica, é possível contribuir significativamente na construção de suas concepções sobre funções reais de forma mais ampla. Consequentemente, acreditamos que as atividades aqui propostas possam contribuir para expandir as imagens de conceito e as relações existentes referentes aos tópicos de funções reais e geometria.

\section{Funções Reais e Geometria Dinâmica}

Para explorar o tema, selecionamos questões da OBMEP cujo enfoque está na relação funcional entre grandezas advindas de problemas intrinsecamente geométricos. O processo será dividido em três etapas: construção de um modelo no Geogebra, representando a situaçãoproblema apresentada na questão; exploração qualitativa do modelo, na qual serão discutidas algumas características da situação-problema e, por fim, a formalização de soluções.

\subsection{Construção de Modelos no Geogebra}

A escolha pelo software Geogebra se justifica pelo fato dele ser um software de geometria dinâmica livre, que "combina geometria, álgebra, tabelas, gráficos, estatística e cálculo numa única aplicação" (AMARAL; FRANGO, 2014, p. 95), com destaque para a possibilidade de interação das representações geométrica e algébrica de um mesmo objeto matemático (REZENDE et al., 2011, AMARAL; FRANGO, 2014). 
O problema inicial é a Questão 18 da $1^{\text {a }}$ fase do nível 3 da OBMEP 2007 (Figura 1). Esta escolha está relacionada ao nosso entendimento de que alunos e professores, ainda que sem conhecimento prévio do software, poderão criar modelos a partir de um breve roteiro, que os oriente na utilização das funções básicas do programa. Os argumentos favoráveis ao uso e à facilidade desse software são variados. Soares (2012, p. 71), por exemplo, afirma que o Geogebra "apresenta uma interface simples, que possibilita ao aluno explorar conceitos de forma dinâmica". Rezende et al. (2011, p. 5) também destacam que "o GeoGebra, com excelente interface dinâmica entre os sistemas algébrico e geométrico de representações, se apresenta como uma poderosa ferramenta para o estudo do comportamento variacional das funções reais".

Note que este é um problema funcional, que relaciona a área do polígono $B C D P$ ao deslocamento do ponto $P$ pela diagonal $A C$. Há algumas ideias importantes na questão que não foram explicitadas no enunciado, a saber, o fato de $A B C D$ ser um retângulo com $A B>B C$.

Um tópico relevante na construção do modelo está exatamente na retomada de conceitos geométricos. O aluno precisa relembrar que se pode construir um retângulo usando retas perpendiculares e paralelas e utilizar este conhecimento para fazer sua construção. No Geogebra, traçar um segmento de reta, retas paralelas, perpendiculares e marcar pontos de interseção são tarefas que não demandam uma curva de aprendizado ${ }^{2}$ longa. Sugerimos que o professor utilize algum tempo para exploração do menu básico: criação de pontos, retas, segmentos, círculos, interseções de objetos, polígonos e ângulos. É de suma importância que, neste primeiro contato com o software, os alunos percebam a estrutura de representação dos elementos, visualizando que para cada objeto construído há um único nome associado a ele na Janela de Álgebra.

Antes da criação do modelo, o professor também pode discutir com seus alunos acerca do que é necessário e suficiente para isto: no problema inicial, por exemplo, basta que se construa um retângulo $A B C D$, a diagonal $A C$, um ponto $P$ sobre a diagonal e o polígono $B C D P$. Sugerimos que os alunos tentem realizar esta construção por si mesmos e apresentamos a seguir uma ideia, sendo que, abaixo de cada instrução, são expostos os botões utilizados para executá-la:

- Na Janela de Visualização 1, criar os pontos $A$ e $B$ sobre o Eixo Horizontal, utilizando o primeiro botão abaixo. Com o segundo botão, criar o segmento $A B$;

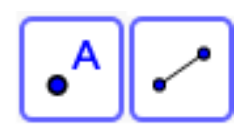

- Criar duas retas perpendiculares ao segmento $A B$, pelos pontos $A$ e $B$;

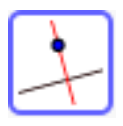

- Marcar um ponto $C$ sobre a perpendicular por $B$ (ferramenta "ponto em objeto" no menu de ponto);

\footnotetext{
${ }^{2}$ Tempo gasto para um iniciante dominar, razoavelmente, as principais ferramentas de um software.
} 


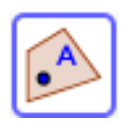

- Por $C$, tirar uma perpendicular à reta $B C$ e encontrar o ponto $D$, na interseção com a outra perpendicular. Para marcá-lo, usar "interseção de dois objetos";

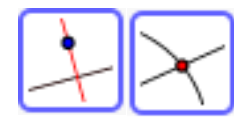

- Com o botão direito do mouse sobre as retas, clicar em "Exibir Objeto" para ocultar as retas. Depois, construir os segmentos $B C, C D$ e $D A$;

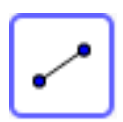

- Construir a diagonal $A C$ e inserir um ponto $P$ (botão direito para renomear) sobre ela.

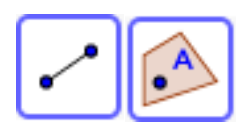

Nesse momento, o retângulo já está pronto. Note que é possível movimentar os pontos $A, B$ e $C$ ( $D$ não, porque foi construído em função dos outros) e verificar que a construção não colapsa, ou seja, o polígono $A B C D$ não se deforma, permanecendo um retângulo. Isto exemplifica o "Princípio da Propriedade Mantida"3, característica das construções dos softwares geométricos.

Para finalizar a construção, falta demarcar a área do polígono BCDP e destacar o segmento $A P$, que será a variável $x$ da questão:

- Com a ferramenta "Polígono", construir $B C D P$, clicando sequencialmente em $B, C, D, P$ e novamente em $B$ para fechar o ciclo;

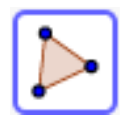

- Com o botão direito sobre o polígono construído, clicar em "Configurações" e "Exibir Rótulo - Valor", para exibir na tela o valor numérico da área do polígono;

- Construir o segmento $A P$, reforçar a espessura e mudar a cor (botão direito, "Configurações", alterar espessura no menu "Estilo" e cor no menu "Cor"). Além disso, nomear como $x$ na legenda e exibir legenda (mesmo menu "Configurações").

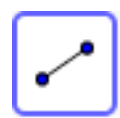

Ao final, teremos um modelo similar ao representado pela Figura 2, no qual movimentandose o ponto $P$ livremente, pode-se verificar o comportamento da área do polígono $B C D P$. Em seguida, apresentaremos a segunda parte da construção, na qual os alunos poderão esboçar o gráfico da função diretamente no Geogebra.

\footnotetext{
${ }^{3}$ Princípio no qual as figuras, em ambientes de geometria dinâmica, devem ser construídas com base em suas propriedades, para que a construção não colapse ao movimentarmos os dados de entrada.
} 
Figura 2 - Tela do Geogebra, Construção Geométrica do Problema 1.

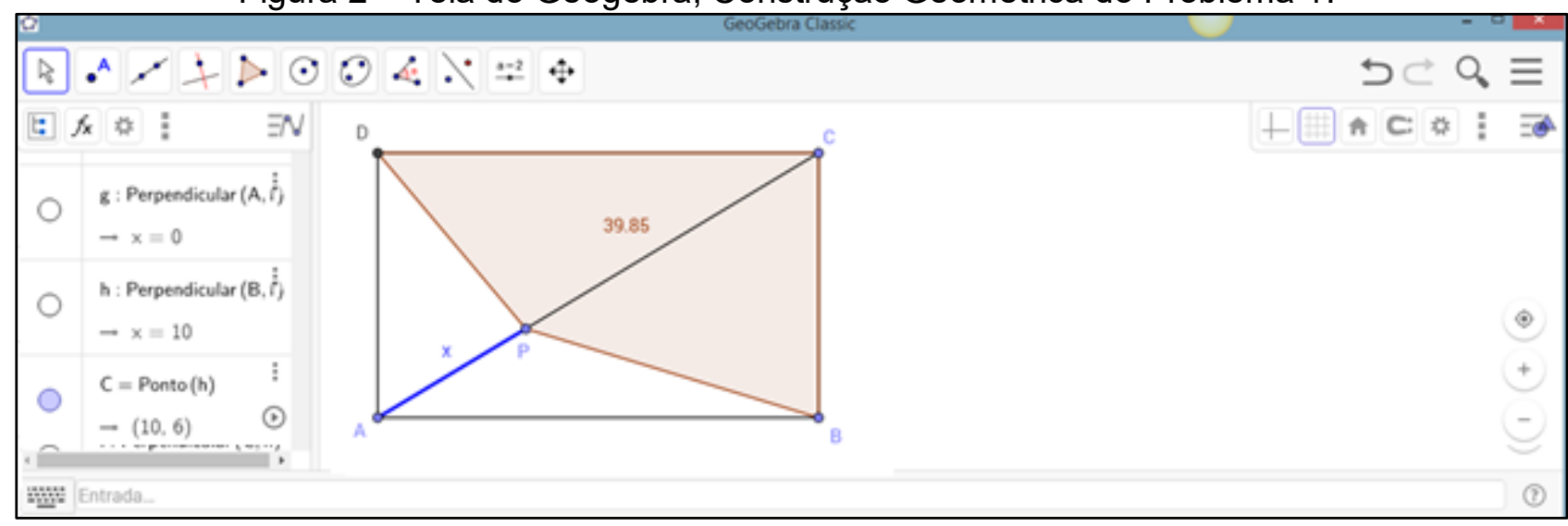

Fonte: Elaboração dos autores.

Deve-se discutir, a priori, quais são os elementos necessários e suficientes para a plotagem do gráfico. Perguntas como "de que depende a área do polígono BCDP?" ou "o que acontece quando o ponto $P$ se desloca sobre a diagonal $A C$ ?" podem conduzir os alunos na percepção de que a variável independente é o segmento $A P$, enquanto a variável dependente é a área de $B C D P$.

Baseados nestas reflexões, é possível justificar que o gráfico da função será descrito por um ponto $Q$ de coordenadas $(x, y)$, tais que $x=A P$ e $y=B C D P$. No Geogebra, procede-se da seguinte forma para a plotagem do gráfico:

- No Menu principal, clicar em "Exibir - Janela de Visualização 2". Aparecerá uma segunda janela gráfica no programa. Ajustá-la para exibir apenas o primeiro quadrante, usando o botão a baixo;

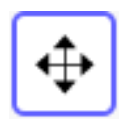

- Olhar, na Janela de Álgebra, os nomes referentes ao segmento $A P$ e ao polígono $B C D P$. No caso da construção apresentada aqui, tomaremos $n$ para $A P$ e $q 1$ para o polígono $B C D P$;

- Clicar na Janela de Visualização 2 e entrar com o seguinte comando na barra de entrada: $Q=(n, q 1)$, em que $n$ é o nome do segmento $A P$ e $q 1$ é a identificação da área do polígono $B C D P$. Ajustar a escala com os botões "Ampliar" e "Reduzir";

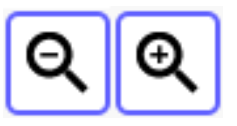

- Movimente o ponto $P$ na Janela 1 e verifique o ponto $Q$ percorrer uma trajetória na Janela 2;

- Com o botão direito sobre o ponto $Q$, clique em "Exibir Rastro". Sobre o ponto $P$, clique em "Animação". Verifique o gráfico gerado pelo ponto $Q$, conforme $P$ se desloca pela diagonal.

Ao final, deve-se chegar a uma construção similar à mostrada na Figura 3. 
Figura 3 - Tela do Geogebra, Construção Geométrica e Gráfica do Problema 1.

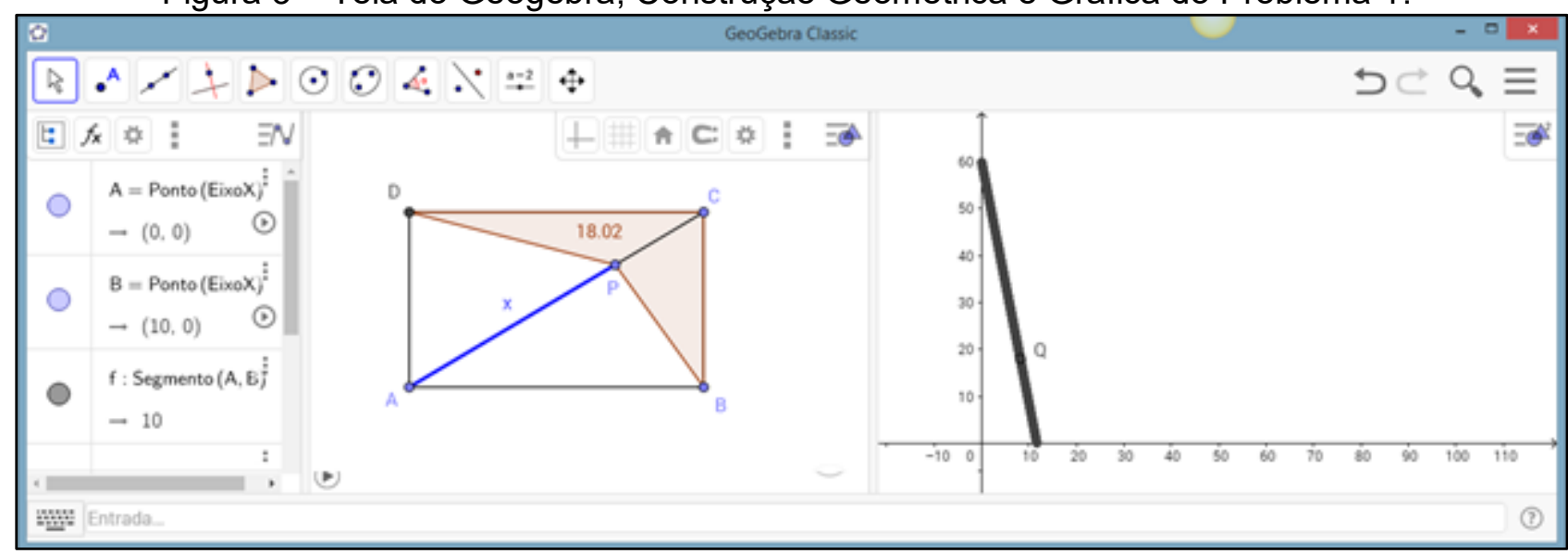

Fonte: Elaboração dos autores.

Note que a simples construção do modelo, no Geogebra, já permite responder que a resposta da questão é o item (B). Esta, por si só, já seria um novo tipo de solução, que chamaremos de solução computacional. Para outros problemas nesta temática, entendemos que este tipo de solução deveria ser estimulada, justamente pelo potencial de retomada de conceitos geométricos básicos, a necessidade de análise sobre os elementos dependentes para a criação do ponto $Q$, que gerará o gráfico, além da possibilidade de exploração de comandos típicos de linguagem de programação (a "Caixa de Entrada" do Geogebra serve como uma linha de comando).

As soluções computacionais ajudam, portanto, a entender melhor os problemas e a visualizar os aspectos funcionais presentes neles, além de nos dar uma resposta totalmente crível do que se pede. A partir daí, não nos interessa mais saber qual é a resposta, mas sim porque esta é a resposta. Note que o foco passa a ser a busca por uma justificativa matemática sobre o que está sendo mostrado no modelo computacional. Isto faz com que os alunos se tornem mais curiosos a respeito da resposta, gerando um ambiente mais propício ao processo ensino-aprendizagem.

\subsection{Exploração Qualitativa dos Modelos e a Busca por Soluções Formais}

Como visto na seção anterior, é possível usar o modelo de, pelo menos, duas formas diferentes: a primeira seria explorar a Janela 1 (Geométrica) antes de montar o gráfico na Janela 2. No entanto, neste cenário ainda estaríamos no mesmo processo de valorizar demasiadamente a resposta correta. Entendemos que a exploração da Janela 2 nos tira o peso de buscar a resposta, deixando-nos apenas na busca de justificá-la. Desta forma, vamos fazer uma análise considerando o modelo totalmente pronto, com as duas janelas de visualização abertas.

O primeiro questionamento a ser discutido está relacionado ao aspecto do gráfico da função. Os professores podem perguntar qual é a natureza deste gráfico e uma resposta esperada é que os alunos respondam que o gráfico é uma "reta decrescente". Não queremos entrar no mérito da linguagem: ao seu próprio tempo, a nomenclatura será ajustada para uma função afim decrescente, fazendo jus à teoria de Imagem de Conceito. O ideal neste momento é relacionar a reta a uma 
variação cuja taxa é constante. Ou seja, se o gráfico é uma reta, então quando percorremos espaços iguais com o ponto $\mathrm{P}$ na diagonal, a variação (neste caso, para baixo) da área do polígono $B C D P$ também deve ser a mesma e isto pode ser explorado com o modelo no Geogebra. Uma sugestão seria utilizar a ferramenta de ponto médio para dividir a diagonal em 4 (ou 8) partes iguais e analisar a variação absoluta da área do polígono. A Figura 4 ilustra este procedimento.

Desta mesma Figura 4, é possível trazer outras conjecturas. Por exemplo:

- Existe uma oposição entre os retângulos II (área 45) e IV (área 15): a parte sombreada no retângulo II seria congruente à parte em branco no retângulo IV;

- A área sombreada é estritamente decrescente, em todo o intervalo do domínio;

- O polígono $B C D P$ pode ser decomposto nos triângulos $B C P$ e $D C P$, de forma que a área de $B C D P$ é a soma das áreas dos triângulos $B C P$ e $D C P$.

Figura 4 - Modelo em 4 momentos: a linearidade no decrescimento da área de BCDP.

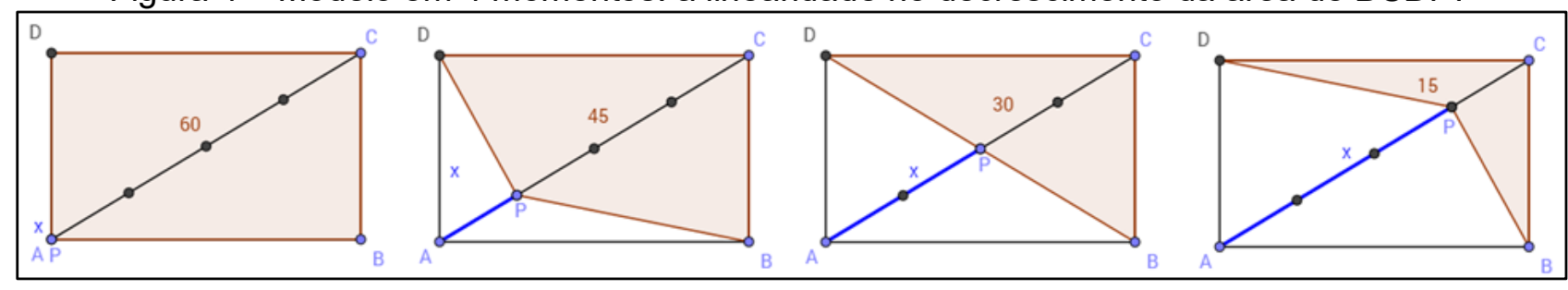

Fonte: Elaboração dos autores.

Por vezes, é comum também que os alunos conjecturem que os triângulos $B C P$ e $D C P$ são congruentes. Pode-se pedir, então, que eles ocultem o polígono $B C D P$ e criem os triângulos $B C P$ e $D C P$ e, movimentando o ponto $P$, vejam o que acontece com as áreas desses triângulos.

Embora a conjectura de que os triângulos sejam congruentes esteja errada ( $A B C D$ é um retângulo com $A B>B C$, logo $D C \neq B C$ ), é verdade que eles são equivalentes. Esta característica pode ser observada com a exploração do modelo e é ela que nos levará a uma solução formal.

Ao refletirmos sobre a equivalência dos triângulos $B C P$ e $D C P$, retomaremos o conceito de área de triângulos: sabemos que esta é dada pelo semiproduto da base pela altura. Desta forma, um caminho viável será observar o que acontece com a base dos triângulos e traçar suas alturas para verificar também o que acontece com elas.

Com o auxílio do modelo (Figura 5), percebe-se que:

1. a base de ambos os triângulos é o segmento $B P$, e a mesma diminui conforme o ponto $P$ se desloca pela diagonal $A C$;

2. as alturas dos triângulos são invariantes ao deslocamento do ponto $P$. 
Figura 5 - Análise das Áreas, Bases e Alturas dos Triângulos BCP e DCP.

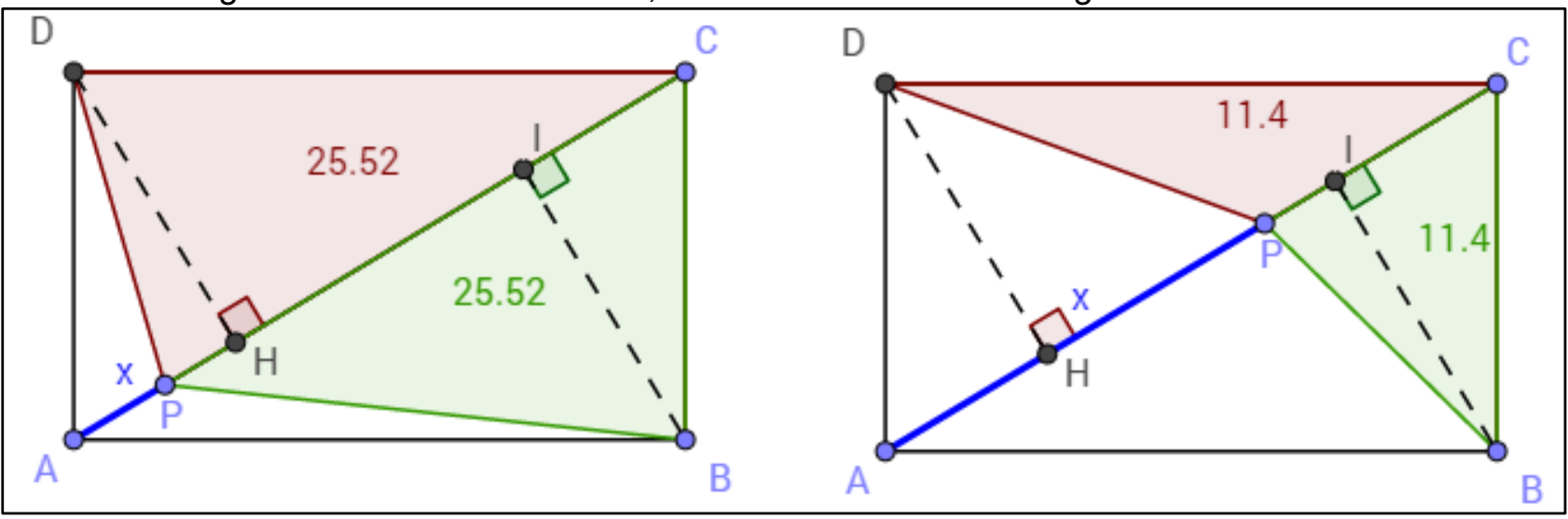

Fonte: Elaboração dos autores.

É fácil ver que as alturas dos triângulos $B C P$ e $D C P$ são congruentes. Para uma justificativa formal, basta perceber que os triângulos $A D C$ e $C B A$ são congruentes ( $L A L)$ e que ambas as alturas de $B C P$ e $D C P$ são alturas homólogas de triângulos congruentes.

A partir desta informação, chega-se a uma nova análise qualitativa da situação-problema: se as áreas dos triângulos $B C P$ e $D C P$ estão em função de suas bases - que decrescem linearmente - e suas alturas, que são invariantes, então estas áreas comportar-se-ão conforme a variação das bases, ou seja, decrescerão linearmente. Justifica-se, portanto, a resposta da situação-problema ser o item (B).

\subsection{Um Segundo Exemplo}

No segundo exemplo (Figura 6), analisaremos outro problema que aborda a temática escolhida. A construção do modelo no Geogebra também não demanda maiores habilidades com o programa, apenas o uso de ferramentas simples:

- Construa os pontos $O, P, Q$ e $R$, de acordo com dados do problema;

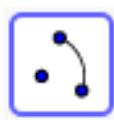

- Trace os segmentos $P Q$ e $R O$ e o arco de círculo $P Q$ (ferramenta "Arco Circular");

- Insira um ponto $M$, sobre $R O$ e construa a corda $A B$, perpendicular a $R O$ por $M$;

- Destaque o segmento $R M$ (legenda: $x$, cor: vermelha; espessura: 5) e crie o quadrado sobre $A B$ (ferramenta "Polígono Regular"). Exiba sua área na tela;

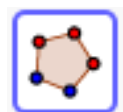

- Na Janela de Visualização 2, crie o ponto $T$, de coordenadas ( $i$,pol1), em que "i" é o nome do segmento em vermelho (cuja legenda é $x$ ) e "pol1" é o nome do quadrado sobre $A B$;

- Habilite o rastro do ponto $T$, anime o ponto $M$ e verifique o programa traçar o gráfico. 
Figura 6 - Um Segundo Exemplo: OBMEP 2009, $1^{\text {a }}$ Fase, Nível 3, Questão 19.

$O$ semicírculo da figura tem centro $O$ e diâmetro $P Q=2 \mathrm{~cm}$. $O$ raio OR é perpendicular a $P Q$. Por um ponto qualquer $M$ de OR traça-se a corda $\mathrm{AB}$ perpendicular a OR. Sejam $x$ o comprimento de RM e $y$ a área do quadrado de lado $\mathrm{AB}$, em $\mathrm{cm}^{2}$.

Qual dos gráficos abaixo expressa a relação entre $x$ e $y$ ?
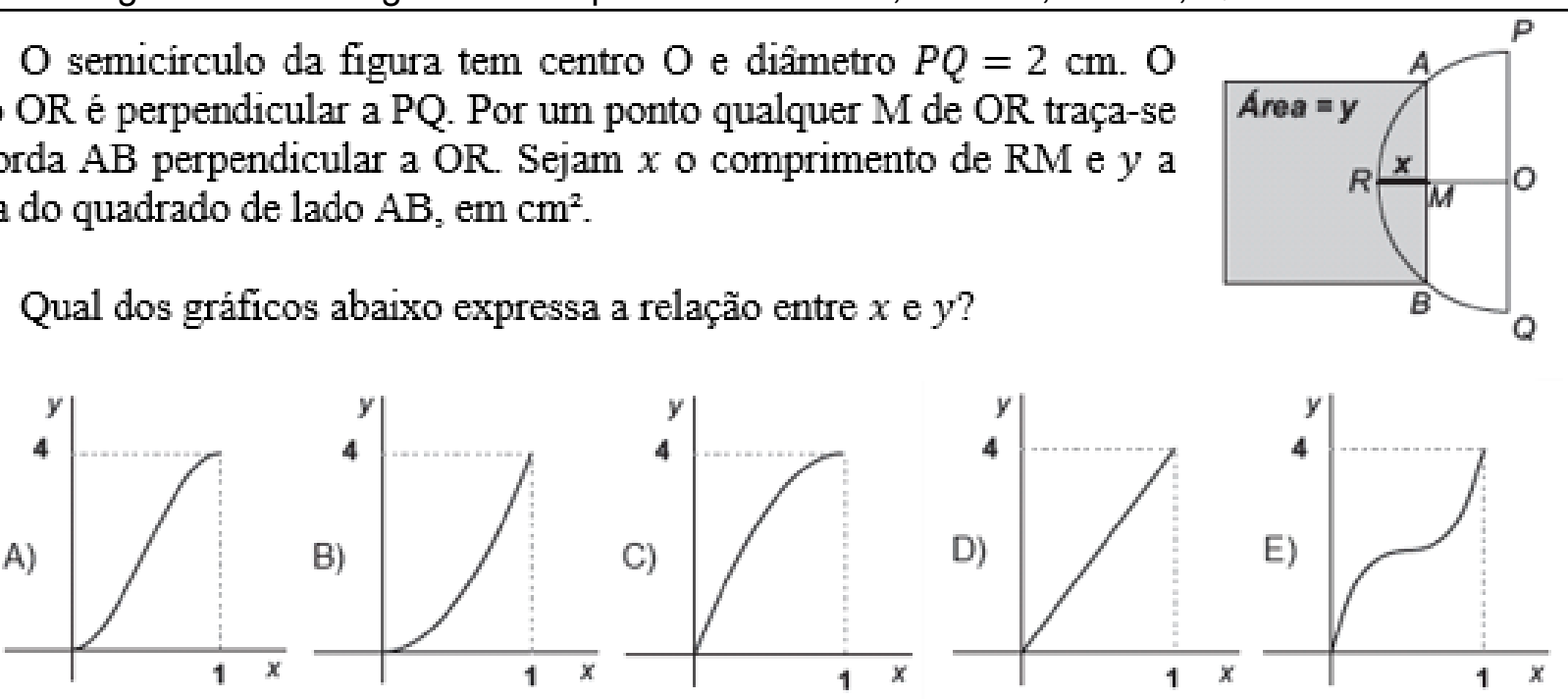

Fonte: OBMEP (2009).

Para esta construção, é interessante utilizar o plano cartesiano, mostrando aos alunos que escolher adequadamente seus pontos de entrada pode ajudar nas construções mais robustas. A ideia aqui é usar o ponto $O=(0,0)$ e, como o diâmetro $P Q$ é igual a duas unidades, teremos $P=(0,1), Q=(0,-1)$ e $R=(-1,0)$. Poderia-se usar também o ponto $R$ como origem, de forma que a medida do segmento $R M$ (destacado em vermelho) se confundiria com a abscissa do ponto $M$. A Figura 7 ilustra o nosso modelo.

Figura 7 - Modelo do Segundo Exemplo: Construção no Geogebra.

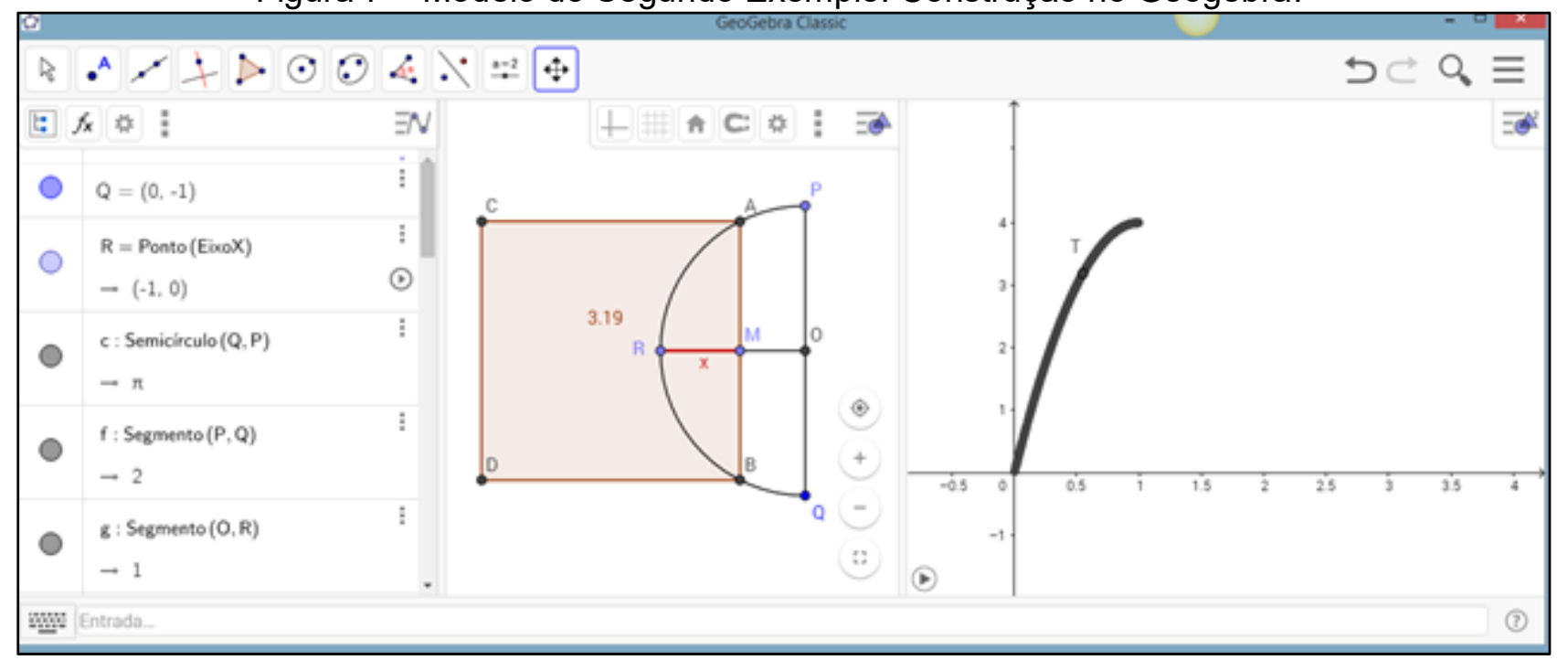

Fonte: Elaboração dos autores.

Uma análise qualitativa da solução computacional, nos permitiria extrair as seguintes informações, dentre outras:

- A função que representa a situação-problema é estritamente crescente;

- Seu gráfico é um ramo de parábola;

- A concavidade da parábola está para baixo. 
Novamente, de posse da resposta do problema, o objetivo é a busca por uma justificativa matemática formal: a figura que nos dá a área pedida é um quadrado e, se o lado $A B$ está aumentando linearmente (de acordo com o deslocamento do ponto $M$ sobre o raio $R O$ ), então o aumento da área não poderá ser linear, pois a área de um quadrado é calculada sobre o produto do lado por ele mesmo. Diferentemente do primeiro problema, aqui há variação dupla, tanto na base quanto na altura do quadrado, de forma que a variação da área obedecerá a uma função polinomial do $2^{\circ}$ grau.

Ao concluir que a função procurada é quadrática, automaticamente temos que seu gráfico deve ser uma parábola. $O$ fato de vermos apenas um de seus ramos pode ser justificado por estarmos tomando o deslocamento de $M$ apenas pelo raio $R O$, não havendo, portanto, uma simetria. Se tomássemos o prolongamento deste segmento e deslizássemos o ponto $M$ até o fim do diâmetro que contém $R$, teríamos a simetria citada e, consequentemente, veríamos o outro ramo da parábola.

Para justificarmos que a concavidade da parábola está para baixo, precisamos refletir sobre a existência de um ponto de máximo na função. Entretanto, esta reflexão já foi feita anteriormente, quando comentamos sobre o deslizamento do ponto $M$ sobre um possível diâmetro. Como a corda $A B$ é limitada pelo círculo, se expandíssemos o ponto $M$ para o prolongamento do raio $R O$, teríamos uma corda $A B$ menor que quando $M$ está sobre $O$, de forma que a área do quadrado seria decrescente, o que nos garante que há um ponto de máximo.

Dito isto, falta uma última discussão: a procura pela expressão algébrica que relaciona domínio e contradomínio. Uma solução natural é perceber que $O A$ é raio (e portanto, tem medida 1 $\mathrm{cm}$ ), enquanto a medida de $O M$ é dada por $1-x$. Sendo o triângulo $A M O$ um triângulo retângulo ( $A B \perp O R$, do enunciado), podemos aplicar o Teorema de Pitágoras para encontrar $A M^{2}=1-(1-x)^{2}=2 x-x^{2}$. Como a área do quadrado é dada por $4 A M^{2}$, segue $S=8 x-4 x^{2}$.

Por fim, apresentamos uma lista com outros problemas da OBMEP nesta temática, em ordem decrescente de edição. Todas as questões destacadas são do Nível 3 (Ensino Médio): 2017 - $2^{\text {a }}$ Fase - Questão 3; 2017 - $1^{\text {a }}$ Fase - Questão 15; 2016 - $2^{\text {a }}$ Fase - Questão 3; 2016 - $1^{\text {a }}$ Fase

- Questão 11; 2015 - 1 ${ }^{\text {a }}$ Fase - Questão 13; 2014 - 2a Fase - Questão 2; 2013 - $2^{\text {a }}$ Fase - Questão 4; 2013 - 1 ${ }^{\text {a }}$ Fase - Questão 12; 2012 - 2 ${ }^{\text {a }}$ Fase - Questão 4; 2011 - 2 ${ }^{\text {a }}$ Fase - Questão 4; 2009 - 2 ${ }^{\mathrm{a}}$ Fase - Questão 5; 2008 - 1 ${ }^{\mathrm{a}}$ Fase - Questão 16; 2006 - $1^{\mathrm{a}}$ Fase - Questão 17.

\section{Considerações Finais}

Atualmente, é cada vez mais relevante o número de pesquisas na área da Educação Matemática que convergem para a necessidade de integração entre os diferentes campos da Matemática no processo ensino-aprendizagem. Neste cenário, o uso de novas tecnologias pode ser tanto um facilitador desta integração, quanto um agente transformador do próprio processo, uma vez que traz novas abordagens acerca dos temas desenvolvidos. 
Ao longo deste artigo, vimos que a criação de modelos computacionais para problemas geométricos-funcionais nos permite desenvolver um novo tipo de solução, a qual chamamos solução computacional. Desta forma, altera-se a dinâmica da investigação matemática, partindo da resposta apresentada pelo recurso computacional para se chegar a uma justificativa formal sobre esta solução. Neste processo, alunos e professores são estimulados a analisarem com maior atenção os diversos aspectos qualitativos das relações funcionais, ao invés de manter um foco excessivo sobre as leis algébricas de formação das funções, ampliando suas imagens de conceito.

A escolha por questões da OBMEP é justificada pelo destaque positivo desta iniciativa presença na quase totalidade dos municípios brasileiros, questões que primam pela destreza do raciocínio lógico-matemático e uma série de iniciativas que visam contribuir para o Ensino de Matemática na Educação Básica4 - e assim acreditamos que a competição possa ser também um norteador para o desenvolvimento do currículo de Matemática na Educação Básica. O recorte pelo tema Geometria e Funções caracteriza-se pelo grau de relevância deste estudo para a Matemática.

Por fim, a utilização de um software como o Geogebra, cuja curva de aprendizado é relativamente simples, pode auxiliar alunos e professores a ingressarem no universo das tecnologias digitais, abrindo caminho para outras produções na área.

\section{Referências}

AMARAL, M. P.; FRANGO, I. Um levantamento sobre pesquisas com o uso do software geogebra no ensino de funções matemáticas. Revista Eletrônica de Educação Matemática, v. 9, n. 1, p. 90-107, 2014.

BIFANO, F. J.; LUPINACCI, L. J. Um binomio dinámico: geometría y funciones. In: La Conferencia Latinoameri-cana de GeoGebra, 2012, Montevideo. Actas.... p. 94-101, 2012.

BRASIL. Parâmetros Curriculares Nacionais: Ciências da Natureza e suas Tecnologias. Brasília: MEC/SEMTEC, 2002.

BRASIL. Base Nacional Comum Curricular. Brasília: MEC/SEB, 2017.

GIRALDO, V. Descrições e conflitos computacionais: o caso da derivada. 2004. $221 \mathrm{f}$. Tese (Doutorado em Engenharia de Sistemas e Computação) - Universidade Federal do Rio de Janeiro, Rio de Janeiro, 2004.

GIRALDO, V. Integrando geometria e funções: gráficos dinâmicos. Revista do Professor de Matemática, v. 79, n. 3, p. 39-46, 2012.

GRAVINA, M. A.; SANTAROSA, L. M. A aprendizagem da matemática em ambientes informatizados. In: IV Congresso RIBIE, 1998, Brasília. Anais... Brasília, p.1-24,1998.

STORMOWSKI, V.; GRAVINA, M. A.; LIMA, J. V. de. Formação de professores de matemática para o uso efetivo de tecnologias em sala de aula. Revista Novas Tecnologias na Educação, v. 13, p. 01-10, 2015.

${ }^{4}$ O Portal da Matemática da OBMEP oferece uma série de recursos (videoaulas, cadernos de exercícios, material teórico, applets, etc.) que abrange conteúdos de Matemática dos Ensinos Fundamental e Médio. 
HARTWING, S. C; PEREIRA, E. C.; MACHADO, C. C.; MIRANDA, S. A. Um olhar sobre as práticas pedagógicas na construção de conhecimentos geométricos. Revista Eletrônica de Educação Matemática, Florianópolis (SC), v. 11, n. 2, p. 243-258, 2016.

HAZZAN, O.; GOLDENBERG, E.P. Students' understanding of the notion of function in dynamic geometry environments. International Journal of Computers for Mathematical Learning, v. 1 , p. 263-291, 1997.

LIMA, E. L; CARVALHO, P. C. P.; WAGNER, E.; MORGADO, A. C. A Matemática do Ensino Médio. v. 1, 6. ed. Rio de Janeiro: Sociedade Brasileira de Matemática, 1996.

LIMA, E. L. Números e Funções Reais. Coleção PROFMAT. Rio de Janeiro: Sociedade Brasileira de Matemática, 2013.

MACHADO, L. Uma Análise Crítica das Provas da 2a Fase da OBMEP. 2015. 109 f. Dissertação (Mestrado Profissional em Matemática em Rede Nacional) - Instituto Nacional de Matemática Pura e Aplicada, Rio de Janeiro, 2015.

CGEE. Centro de Gestão e Estudos Estratégicos. Avaliação do impacto da Olimpíada Brasileira de Matemática nas escolas públicas - OBMEP 2010. Série Documentos Técnicos. $n$. 11, julho 2011.

MENEGHETTI, R. C. G.; REDLING, J. P. Tarefas Alternativas para o Ensino e a Aprendizagem de Funções: análise de uma intervenção no Ensino Médio. Boletim de Educação Matemática, v. 26, n. 42A, p. 193-229, 2012.

MEZA, E.S.; SCHIRLO, A. C. Relendo a OBMEP. In: Congresso Nacional de Educação EDUCERE, 9, 2013, Curitiba. Anais... EDUCERE, 2013.

NEVES, J. D.; RESENDE, M. B. O proceso de ensino-aprendizagem do conceito de função: um estudo na perspectiva da teoria histórico-cultural. Educação Matemática Pesquisa, v. 18, n. 2, p. 599-625, 2016.

PAVANELLO, R. M.; NOGUEIRA, C. M. I. Avaliação em Matemática: algumas considerações. Estudos em Avaliação Educacional, v. 17, n. 33, p. 29-41, 2006.

OBMEP. Prova da $1^{\text {a }}$ Fase do Nível 3. 2007. Disponível em: <http://www.obmep.org.br/provas static/pf1n3-2007.pdf>. Acesso em: 30 ago. 2018.

OBMEP. Prova da $1^{\text {a }}$ Fase do Nível 3. 2009. Disponível em:

<http://www.obmep.org.br/provas static/pf1n3-2009.pdf>. Acesso em: 30 ago. 2018.

OBMEP. Provas e soluções. Disponível em: <www.obmep.org.br/provas.htm>. Acesso em: 30 ago. 2018.

REZENDE, W. M; PESCO, D. U; BORTOLOSSI, H. J. Explorando aspectos dinâmicos no ensino de funções reais com recursos do GeoGebra. In: Conferência Latino-Americana de Geogebra, 1, 2011, São Paulo. Anais... GEOGEBRA - LA, 2011.

SANTOS, G. L. D.; BARBOSA, J. C. Como ensinar o conceito de função? Educação Matemática em Revista, v. 53, p. 27-37, 2017.

SOARES, L. H. Tecnologia computacional no ensino de matemática: o uso do Geogebra no estudo de funções. Revista do Instituto Geogebra Internacional de São Paulo, v. 1, n. 1, p. 6680, 2012. 
SOARES, C. M. M; LEO, E.; SOARES, J. F. Impacto da Olimpíada Brasileira de Escolas Públicas (OBMEP) no desempenho em matemática na Prova Brasil, ENEM e PISA. 2014. Disponível em: <http://server22.obmep.org.br:8080/media/servicos/recursos/420951.o>. Acesso em: 30 ago.2018.

TALL, D.; VINNER, S. Concept image and concept definition in mathematics, with special reference to limitis and continuity. Educational Studies in Mathematics, n. 12, p. 151-169, 1981. 